\section{For patients with dry mouth}

Molar Ltd has announced the arrival of new bioXtra Sucking Tablets to complement the bioXtra range of products. bioXtra is designed specifically for patients who suffer from dry mouth (xerostomia).

This extremely unpleasant condition can result in patients having difficulty speaking, eating and sleeping and can be detrimental to their oral health. The range has been designed to provide patients with a better quality of life, providing comfort, salivary stimulation and hygiene.

The new Sucking Tablets have a mild flavour to give a cooling effect in the mouth and to help stimulate saliva flow. The active ingredients include xylitol and lactoperoxidase and lactoferrin which are derived from colostrum extract. The recommended price is $£ 4.95$ for a pack of 60 tablets.

Other products include Mild Antibacterial Toothpaste and Alcohol-free Mouth rinse for daily hygiene, Nonstick Dental Gum and the New Sucking Tablets for salivary stimulation and Moisturising Gel and Moisturising Gel Mouth spray (Both NHS prescribed) for long-lasting comfort.

Reader response number 51

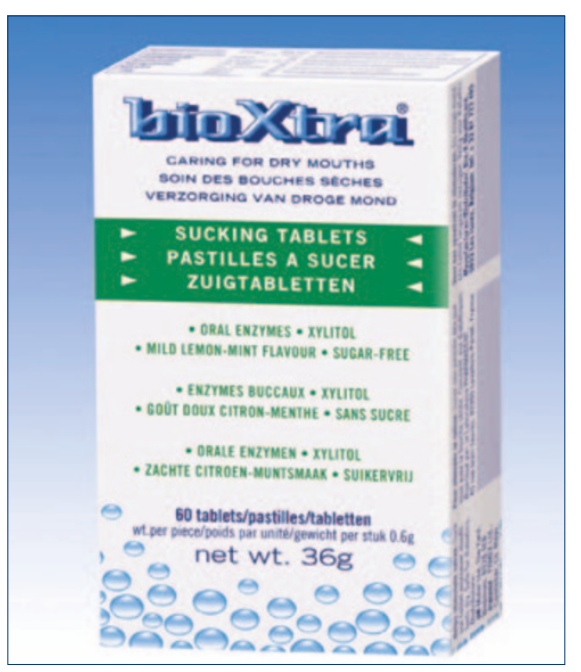

\title{
Total patient contact solution
}

A new total patient call system has been launched by healthcare messaging solutions company iPlato, in partnership with Biomni Voice, a provider of automated telephone voice messaging and reminder services.

The new integrated solution means that patients can be automatically contacted by either landline or mobile phone to significantly reduce Did Not Attend (DNA) rates for dental appointments.

Biomni Voice messaging service works by allowing appointment reminder messages to be created and converted to natural sounding speech or by selecting a pre-recorded message using real voice.

The voice service prompts the call recipient to respond immediately via their telephone keypad. All calls and responses are logged and reported to enable appropriate action to be taken when needed. The service will also retry

\section{Portable and lightweight}

The new WisLight II gives you the choice of fast and effective tooth whitening with far less sensitivity. Choose from LED or Pulsation Technology from one simple to use portable, lightweight unit.

The WisLight II is a combined unit that allows you to use the same system whichever option you choose to use. The LED provides high concentration and quick whitening; the Pulsation option is used for low concentration along with fast custom tray whitening. Both provide swift, safe and effective long-term dual arch whitening in around 10-15 minutes.

The WisLight II is completely portable (battery powered is an option), and uses rechargeable batteries (option), however if you do run out of power you can use a mains attachment to give greater flexibility. This cordless system allows the WisLight II to be quickly and easily at pre-determined intervals and detect answer machines or voicemail.

Reader response number 50

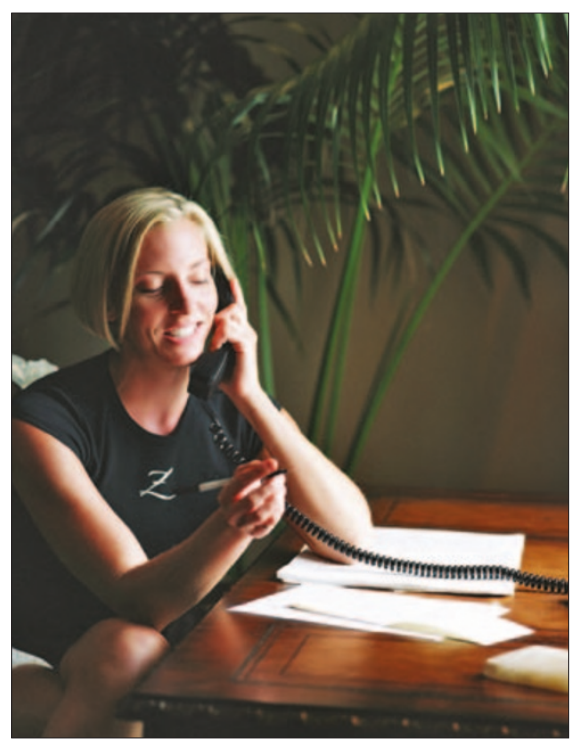

wheeled into different surgeries within the practice. It is supplied complete with two pairs of safety glasses, four cheek retractors, whitening head, pulsation head, power adapter and complete instruction manual.

Reader response number 52

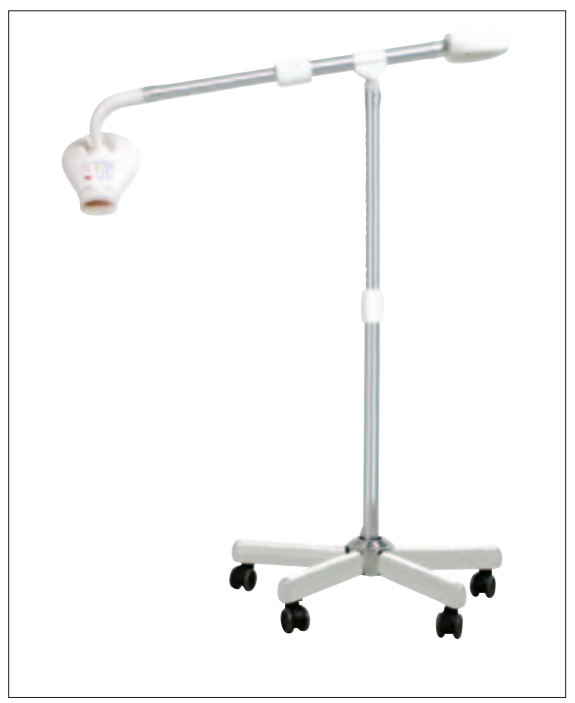




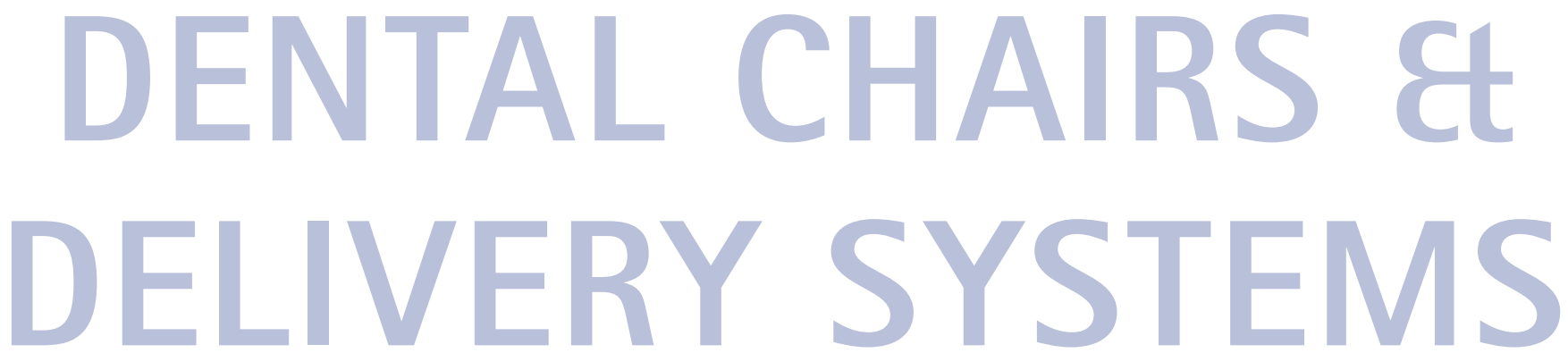

\section{Reduce risk of neck and back pain}

The DynamoSeat and InCharge stools from Evident are designed for all members of the dental team. DynamoSeat and InCharge stools automatically correct your seating position creating perfect balance no matter what height you may be working at, reducing the risk of neck and back pain and enabling you to work at a comfortable distance from the patient.

The stool's soft castors suit all types of flooring, giving full mobility and support to practitioners at the chairside. The washable and easy to remove fabric seat covers ensure cleanliness and hygiene, and the stools are available in a wide variety of styles and colours, allowing you to choose stools that complement your working conditions and the function and interior design of your practice. Reader response number 55

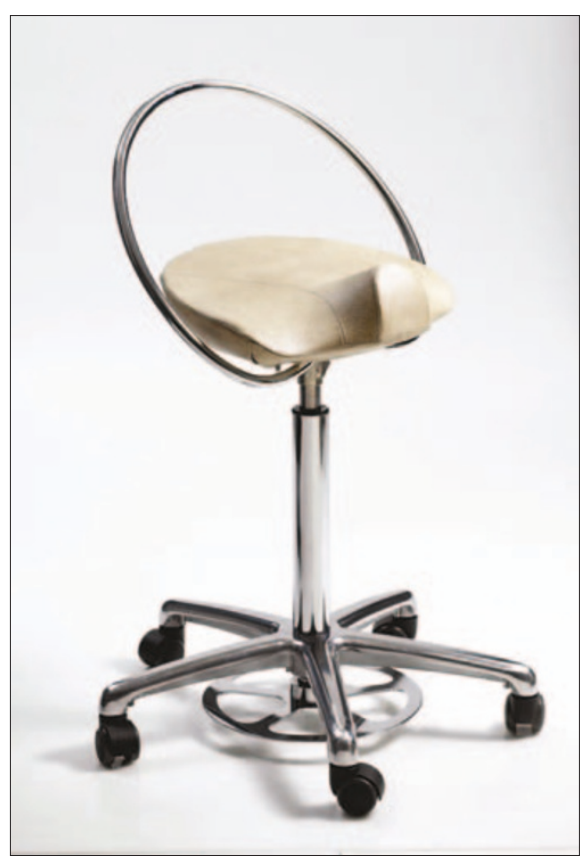

\section{Easily integrated unit}

The Cavitron OEM system delivers the same SPS performance of other Dentsply systems (maintaining clinical power at lower settings even with heavy calculus build-up) in a compact box that easily integrates into your delivery unit.

This built-in unit accepts your existing $30 \mathrm{~K}$ Cavitron Inserts and also comes with a detachable hand piece that can be autoclaved, helping reduce the risk of contamination. The Blue Zone gives an extended low power range increasing your patient comfort, especially during sub-gingival scaling. The

\section{Pain free suction}

Trycare Dental Linkline's general catalogue features the SDI Directa Adaptapal Suction Adapter, a simple solution to pain free suction. The product eliminates the need to manoeuvre heavy, inflexible suction hoses.

Bending easily, Adaptapal enables the operator to get good access from all ultrasonic scaler can be installed into any dental unit.

Reader response number 53

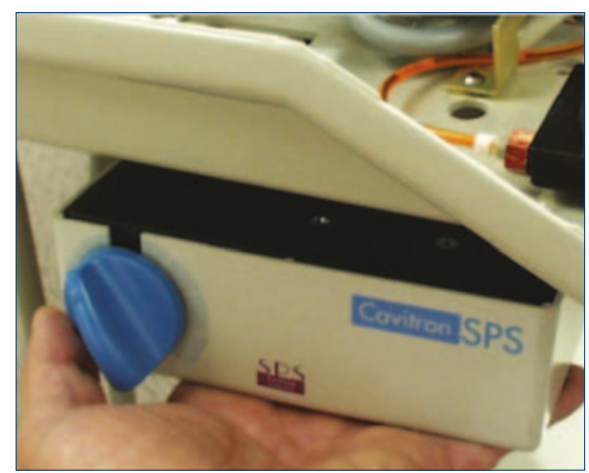

angles facilitating better control. Easy to remove for cleaning, Adaptapal also serves as a barrier shield because you hold it rather than the aspirator hose.

Available in two sizes (blue $6.3 / 11 \mathrm{~mm}$ and yellow 11/11 mm), Adaptapal is supplied in packs of four. It can be disinfected in washer disinfectors or autoclaved up to $120^{\circ} \mathrm{C}$ and can be used up to 30 times.

Reader response number 54

\section{Chairside ceramic restorations}

The Cerec 3D system from Sirona Dental Systems can help you produce chairside ceramic restorations.

The system allows you to place the new prosthesis in the same visit saving you and your patient time and laboratory fees.

After taking an optical impression of the preparation and the antagonist, you are in complete control of the finished prosthesis. You specify the positions of the margins and the proximal contacts.

From there on the Cerec system fabricates the crown, inlay or onlay accurately, quickly and to the highest quality.
Cerec automatically accurately computes the occlusal contacts, referring to the antagonist to do so.

Reader response number 56

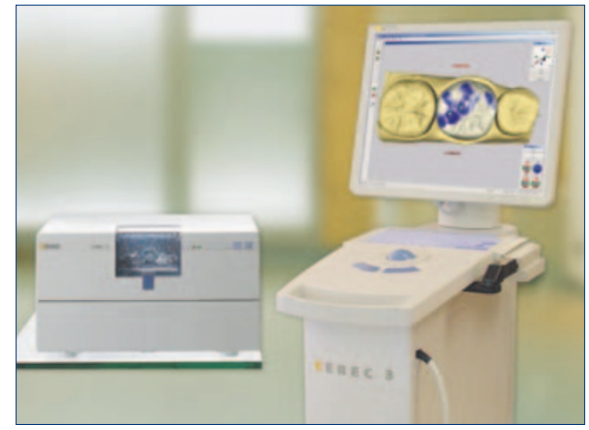




\section{Gas-lift height adjustment}

Stevens Seating Limited offer a range of stools suitable for the surgery. The Model $\mathrm{B}+$ and Model $\mathrm{A}+$ are the latest versions of the Pratique stool.

The Model A+ assistants stool has an 18 inch small aluminium base, is silver finished with an adjustable backrest height. The Model B+ operators stool has quality dual-wheeled black castors, durable vinyl fabric, gas-lift height adjustment and comes in a wide range of colours. Lastly, the Model C is constructed from quality components with instant left or right-handed gas-lift height adjustment. Reader response number 57

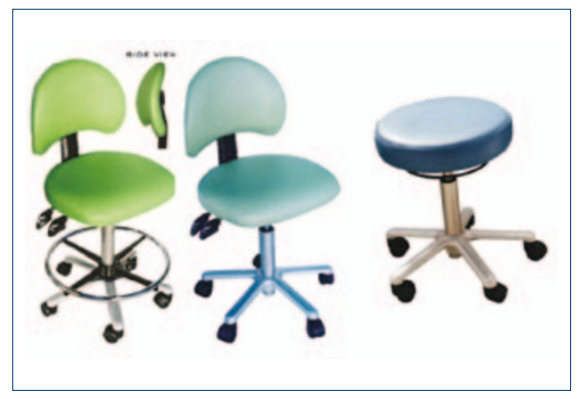

\section{Create an individual practice}

Sirona have launched a new design collection that enables dentists to style their practices to their own personal tastes and also create one that will make their patients feel relaxed and well looked after.

The new Sirona Collection offers three

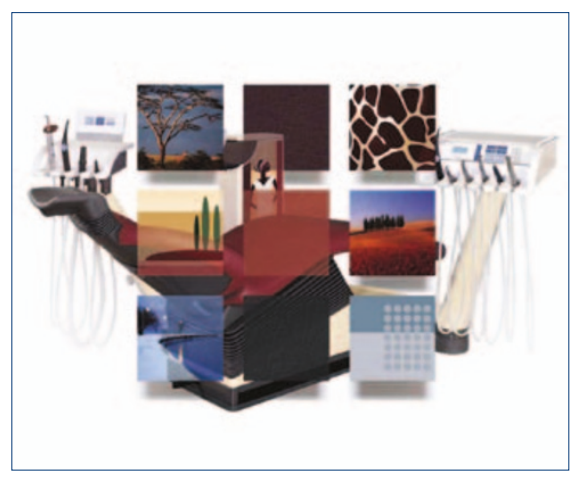

attractive themes: Savannah, Polar and Mediterranean. These ambiences allow the dentist to work in the warmth of the African sun, the cool of Polar nights or the tranquil beauty of Mediterranean landscapes. The new Sirona Collection incorporates many different combinations, which are available for all models in the Sirona treatment centre range.

Each of the designs offers different combinations of upholstery colours, paintwork colours and themed graphics on the decor panels of the water unit. To help dentists pick the perfect combination Sirona have created a colour configurator online at www.sirona.com/ colourconfigurator. The dentist can see at a glance what the treatment centre will look like in situ.

Reader response number 58

\section{Design and functionality combined}

Tavom's range of dental practice furniture and cabinetry includes the latest addition to its product portfolio, the Alnair range, which offers the ultimate in appearance, simplicity in maintaining hygiene standards and genuine functionality.

The surgery suite is available in a wide variety of colours to brighten any dental practice, and at the same time offers dental professionals value for money and durable, versatile, hardworking work surfaces.

In spite of its emphasis on colour and style, the Alnair range is particularly easy to clean, with rectangular sinks integrated into the worktops for an elegant appearance.

All Tavom's products are manufactured in a state of the art facility in Italy.

Reader response number 59

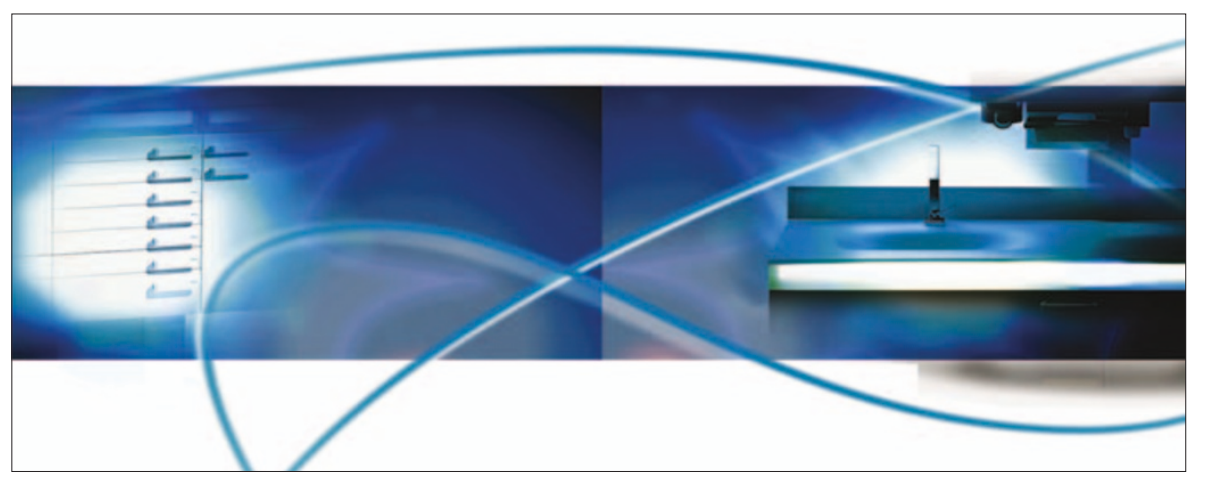

